\title{
Screening phytochimique, dosage des polyphénols et détermination de l'activité antioxydante de deux plantes anti- hypertensives du Niger
}

\author{
Zakari Seybou Djamilatou, PhD \\ Dr. Alfa Keita Djibo \\ Dr. Bakasso Sahabi \\ Dr. Sabo Haoua Seini
}

Laboratoire des Substances Naturelles et de Synthèse Organique (LASNASO), Université Abdou Moumouni de Niamey, Niger

Doi:10.19044/esj.2021.v17n17p335

Submitted: 18 February 2021

Accepted: 21 April 2021

Published: 31 May 2021
Copyright 2021 Author(s)

Under Creative Commons BY-NC-ND

4.0 OPEN ACCESS

Cite As:

Djamilatou Z.S., Djibo A.K. Sahabi B. \& Seini S.H. (2021). Screening phytochimique, dosage des polyphénols et détermination de l'activité antioxydante de deux plantes anti-hypertensives du Niger. European Scientific Journal, ESJ, 17(17), 335.

https://doi.org/10.19044/esj.2021.v17n17p335

\section{Résumé}

Acanthospermum hispidum Dc. et Ximenia americana L. sont largement utilisées en Afrique subsaharienne pour le traitement traditionnel de plusieurs pathologies, dont l'hypertension artérielle. L'objectif du présent travail est de déterminer l'activité antioxydante des extraits bruts de ces plantes, ainsi que les molécules qui leurs confèrent ces propriétés. A cet effet, les parties aériennes d'A. hispidum récoltées à Niamey et l'écorce de tronc de $X$. americana récoltée à Gaya ont été extraites avec des solvants de polarité croissante, respectivement l'hexane, l'acétate d'éthyle, l'éthanol et le méthanol. Il s'en est suivi le screening phytochimique, le dosage des polyphénols, des flavonoïdes et la détermination des activités antioxydantes. Il est ressorti des résultats du screening phytochimique par des réactions en tubes, la présence des coumarines, des tanins, des flavonoïdes, des stérols et triterpènes, des saponosides, des oses et holosides dans les deux plantes. Le dosage des polyphénols totaux par la méthode du Folin- Ciocalteu a montré que les teneurs en polyphénols totaux varient de 0,627 à 1,583 mg d'équivalent acide gallique (EqAG) pour A. hispidum et de 0,036 à 1,645 mg d'EqAG pour 
$X$. americana. Le dosage des flavonoïdes avec $\mathrm{AlCl}_{3}$ a montré que tous les extraits contiennent des flavonoïdes mais que c'est particulièrement les extraits AcOEt d' A. hispidum et EtOH de X. americana qui sont les plus riches avec des taux respectifs de 0,089 $\mathrm{mg}$ et $0,069 \mathrm{mg}$ EqQ. L'extrait EtOH de l'écorce de tronc de $X$. americana a montré la plus grande capacité antiradicalaire $(129,56 \%)$. Cette activité élevée pourrait être due aux teneurs élevées en flavonoïdes et en polyphénols totaux. L'effet de ces molécules sur les radicaux libres semblerait être à l'origine de l'activité antihypertentive des plantes étudiées.

Mots clés : A. hispidum, X. americana, polyphénols, activité antioxydante, hypertension, Niger

\title{
Phytochemical Screening, Dosage of Polyphenols, Flavonoids and Antioxydant Activity of Two Anti-Hypertensive Plants from Niger
}

\author{
Zakari Seybou Djamilatou, PhD \\ Dr. Alfa Keita Djibo \\ Dr. Bakasso Sahabi \\ Dr. Sabo Haoua Seini
}

Laboratoire des Substances Naturelles et de Synthèse Organique (LASNASO), Université Abdou Moumouni de Niamey, Niger

\begin{abstract}
Acanthospermum hispidum Dc. and Ximenia americana L. are widely used in sub-Saharan Africa in the traditional treatment of several pathologies, including hypertension. The main objective of this work is to determine the antioxidant activity of the crude extracts of these plants, as well as the molecules that confer these properties. For this purpose, the aerial parts of $A$. hispidum from Niamey and the stem bark of $X$. americana from Gaya were extracted with solvents of increasing polarity, respectively hexane, ethyl acetate, ethanol and methanol. Phytochemical screening, dosage of polyphenols and flavonoids, and the determination of the antioxidant activities of these extracts followed. The results of the preliminary phytochemical screening showed the presence of coumarins, tannins, flavonoids, sterols and triterpens, saponosides, oses and holosides in both plants. The Folin ciocalteu method showed that the total polyphenol contents of these same extracts ranged from 0.627 to $1.583 \mathrm{mg}$ of gallic acid equivalent (AG/Eq) for $A$. hispidum and from 0.036 to $1.645 \mathrm{AG} / \mathrm{Eq}$ for $X$. americana. Total flavonoid
\end{abstract}


assay with $\mathrm{AlCl}_{3}$ showed that all extracts contain flavonoids but that it is particularly the extracts ethyl acetate (AcOEt) of A. hispidum and ethanol $(\mathrm{EtOH})$ extract of $X$. americana that are the richest with respective levels of $0.089 \mathrm{mg}$ and $0.069 \mathrm{mg} \mathrm{EqQ}$. The EtOH extract of the trunk bark of $X$. americana showed the greatest antiradical capacity (129.56\%). This high activity could be due to the high content of flavanoids and total polyphenols. The effect of these molecules on free radicals seems to be at the origin of the antihypertensive activity of the plants studied.

Keywords: A. hispidum, X. americana, polyphenols, antioxidant activity, hypertension, Niger

\section{Introduction}

L'utilisation des plantes médicinales est très ancienne et connaît un regain d'intérêt aussi bien pour les populations que pour les scientifiques, notamment dans les pays en voie de développement où les systèmes de santé sont peu développés et limités aux grandes villes. Les plantes médicinales possèdent diverses activités biologiques et pharmacologiques. Elles sont des réservoirs d'une grande variété de métabolites secondaires : les alcaloïdes, les composés phénoliques et les terpénoïdes (Zerargui et al., 2015). Parmi ces métabolites, les composés polyphénoliques sont des sujets de recherche brûlants dans le monde entier actuellement en raison de leurs activités physiologiques et pharmacologiques (Juanying et al., 2019). Plus de 50 spécialités contenant un ou plusieurs polyphénols sont disponibles en France. Ils sont regroupés dans la catégorie des veinotoniques et des vasculoprotecteurs. Parmi les veinotoniques, on peut citer le Relven ou le Cirkan renfermant du ruténoside, le Daflon ou le Diosmilt renfermant de la diosmine et le Vasococitrol ou le Cemaflavone renfermant des citroflavonoïdes (Cyril et Valérie, 2014). En effet les composés phénoliques possèdent diverses activités biologiques dont le potentiel antioxydant contre le processus oxydatif induit par les espèces réactives à l'oxygène (ERO). Ces espèces (ERO) peuvent être des radicaux libres tels que l'anion superoxyde, des radicaux hydroxyles et des espèces non radicalaires telles que le peroxyde d'hydrogène, l'oxygène singulet (Inbathamizh.et al., 2013). Les lipides, l'ADN et les protéines sont les principales cibles des ERO dans l'organisme (Lobo. et al. 2010). En effet, le stress oxydatif provoqué par l'excès des ERO non contrôlés est impliqué dans plusieurs pathologies, notamment l'hypertension, l'hypercholestérolémie, le diabète, l'insuffisance cardiaque(Leong et Shui, 2002; Gönenç et al., 2013), l'athérosclérose, l'ischémie-reperfusion, la maladie d'Alzheimer, l'arthrite rhumatismale, le cancer ,vieillissement (Leong et Shui, 2002; Inbathamizh et al., 2013), les maladies neurodégénératives (Leong et Shui, 2002), les troubles immunologiques et les inflammations chroniques ( 
Ramana et al., 2013). La consommation d'aliments riches en polyphénols réduit le développement de nombreuses pathologies, telles que le cancer, l'ischémie cardiaque, l'athérosclérose et l'hypertension (Hertog et al., 1999, Hertog et al., Middleton et al., 2000, Leong et Shui, 2002).

L'hypertension artérielle(HTA) constitue un véritable problème de santé publique dans le monde en général et en particulier au Niger. L'enquête de l'OMS pour la surveillance des facteurs de risques des maladies chroniques, réalisée au Niger a révélé une prévalence pour l'HTA à 21,2\% chez les adultes. Au terme de l'étude de Hassimi et al, 2013, il ressort une forte prévalence de 1'HTA $(61,61 \%)$ avec un retentissement cardiaque chez les patients en hémodialyse chronique à l'hôpital national lamordé de Niamey. Zeinabou et al., 2016 ont rapporté que les signes cliniques à l'admission de $86,6 \%$ de patients en insuffisance rénale aigue obstétricale à la maternité Issaka de Gazoby de Niamey étaient hypertendus. Cependant la prise en charge de l'HTA par les médicaments conventionnels est un traitement à vie et demeure toujours couteuse amenant plusieurs patients à s'orienter vers la médecine traditionnelle qui constitue un moyen de soins primaires. Pourtant des produits ayant l'innocuité, l'efficacité et la qualité prouvées doivent être utilisés. Au regard des effets bénéfiques affectés aux polyphénols, et de l'ampleur de l'hypertension artérielle au Niger, on s'est fixé comme objectif à travers cette étude d'estimer la teneur en polyphénols et d'évaluer le pouvoir antioxydant de Acanthospermum hispidum DC. et Ximenia americana L. deux espèces de la pharmacopée nigérienne utilisées dans la prise en charge traditionnelle de cette pathologie.

\section{Materiel et Methodes Materiel}

Nous avons porté notre choix sur A. hispidum DC. et X. americana L., après une enquête ethnobotanique couplée avec une étude bibliographique des plantes recensées chez les tradipraticiens de Niamey. Les sites de récolte ont été ciblés avec l'aide d'un guide (technicien en botanique de la Faculté des Sciences et Techniques de l'Université Abdou Moumouni).

Le matériel végétal est constitué de la partie aérienne d'A. hispidum récoltée le 07/08/2018 à Niamey et de l'écorce de tronc de $X$. americana $\mathrm{L}$. récoltée à Gaya le 14/08 2018. Il a été identifié au département de biologie de l'université Abdou Moumouni de Niamey par le professeur Sadou MAMANE. Les parties récoltées ont été séchées sous ventilation permanente pendant deux semaines puis pulvérisées à l'aide d'un broyeur électrique (Marque RETSCH, Type SK 100). 


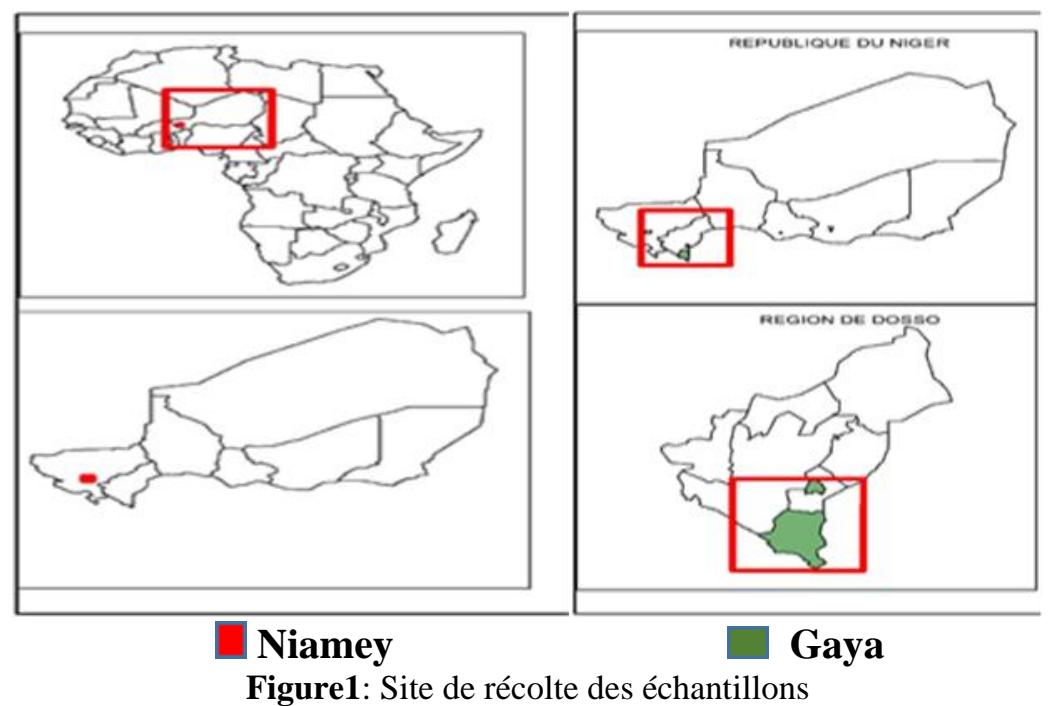

\section{Methodes}

\section{Etudes phytochimiques}

Les grandes familles de métabolites secondaires ont été recherchées par des réactions colorées en tube suivant les méthodes classiques de caractérisation sur la poudre des plantes étudiées. Les tanins et polyphénols ont été identifiés par le test au $\mathrm{FeCl}_{3}$ et le réactif de Stiasny; les flavonoïdes par la réaction à la cyanidine; les saponosides par le test de mousse ; les triterpènes et stéroïdes par le test de Liebermann-Burchard et enfin les alcaloïdes par les tests de Mayer (Brunetton J.1999 ; Evans, 2002).

\section{Extraction des polyphénols}

Une extraction des polyphénols a été réalisée avec un appareil Soxhlet en utilisant des solvants de polarités croissantes. Le ratio solideliquide est de $1: 100(\mathrm{~m} / \mathrm{v}) .2,5 \mathrm{~g}$ de chaque échantillon a été chargé dans une cartouche de cellulose $(22 \mathrm{~mm} \times 80 \mathrm{~mm})$ et extrait avec $250 \mathrm{ml}$ de solvant. Chaque échantillon a été dégraissé d'abord avec de l'hexane (30 min par cycle) pendant cinq cycles et extrait successivement avec l'acétate d'éthyle (35 min par cycle), de l'éthanol (40 min par cycle) et du méthanol (50 min par cycle) pendant 10 cycles par solvant. Les fractions obtenues ont été réunies, filtrées puis concentrées avec un évaporateur rotatif sous vide jusqu'à un volume final de $25 \mathrm{ml}$ et conservé dans un flacon en verre au réfrigérateur jusqu'à utilisations ultérieures. Les extraits concentrés ont été utilisés pour déterminer les teneurs en composés phénoliques totaux (TCPT), en flavonoïdes totaux (FT) et la capacité d'élimination des radicaux libres (Kannika et al., 2018). 


\section{Détermination de la teneur en composés phénoliques totale (TCPT)}

La teneur en composés phénoliques totaux (TCPT) a été déterminée avec le réactif de Folin-Ciocalteu (Cicco et al., 2009). $100 \mu$ d'extrait brut ou standard a été mélangé avec $500 \mu \mathrm{l}$ de réactif Folin-Ciocalteu à 10\% (v / v). Le mélange a été placé dans l'obscurité pendant 3 min avant l'ajout de $400 \mu \mathrm{l}$ de 7,5\% (p/v) de $\mathrm{Na}_{2} \mathrm{CO}_{3}$. Le mélange a été incubé dans l'obscurité pendant $30 \mathrm{~min}$ et l'absorbance a été mesuré à $731 \mathrm{~nm}$ en utilisant un spectromètre (KONTRON instruments, type : UVIKON $922 \mathrm{~A}$ ). La mesure a été comparée à une courbe standard préparée avec 10, 20, 40,60, 80 et $100 \mathrm{mg} / 1$ d'acide gallique (AG). La TCPT a été exprimée en équivalent acide gallique(EqAG).

\section{Détermination de la teneur en flavonoïdes (TF)}

La teneur en flavonoïdes a été calculée par la méthode décrite par (Dirar et al.; 2019). Cette méthode consiste à ajouter $25 \mu \mathrm{L}$ d'extrait, $75 \mu \mathrm{L}$ d'éthanol. Puis $5 \mu \mathrm{L}$ d'une solution d' $\mathrm{AlCl}_{3}$ (10\% dans le méthanol) et $140 \mu \mathrm{L}$ d'eau distillée ont été ajouté. Le mélange est agité et laissé à la température ambiante pendant $30 \mathrm{mn}$. L'absorbance a été mesurée contre un blanc préparé sans $\mathrm{AlCl}_{3}$ à $420 \mathrm{~nm}$. La teneur en flavonoïdes totaux est calculée en termes d'équivalent quercétine $(\mathrm{EgQ})$ par référence au courbe étalonnage tracée avec 10, 20, 40,60, 80 et $100 \mathrm{mg} / 1$ de quercétine.

\section{Détermination de l'activité antioxydante in vitro}

L'activité antioxydante a été évaluée avec le 2,2-diphényl-1 picrylhydrazyle (DPPH) (Brand-Williams et al., 1995). $20 \mu$ d'extrait brut a été mélangé à $180 \mu \mathrm{l}$ d'une solution à $80 \mu \mathrm{M}$ de DPPH dissout dans le méthanol. Le mélange a été placé dans l'obscurité pendant $30 \mathrm{~min}$. Ensuite, l'absorbance a été lue à $515 \mathrm{~nm}$. L'activité anti-oxydante de l'extrait a été calculée à l'aide des équations suivantes :

Activité de piégeage des radicaux libres $(\%)=\left\{\left(\mathrm{A}_{0}-\mathrm{A}_{1}\right) / \mathrm{A}_{0}\right\} \times 100$

$\mathrm{A}_{0}$ et $\mathrm{A}_{1}$ sont les valeurs de l'absorbance du blanc et de l'échantillon d'essai, respectivement. 


\section{Resultats et Discussion \\ Screening phytochimique}

Tableau 1 : Résultats du screening phytochimique de la partie aérienne de A. hispidum et l'écorce tronc de $X$ americana

\begin{tabular}{|c|c|c|}
\hline \multirow{2}{*}{ Groupes chimiques } & \multicolumn{2}{|c|}{ Espèces } \\
\cline { 2 - 3 } & A. hispidum & X. americana \\
\hline Alcalö̈des & - & - \\
\hline Caroténoïdes (Carr and Price) & ++ & - \\
\hline Coumarines (UV 366) & ++ & ++ \\
\hline Anthracénosides libres & - & ++ \\
\hline Anthracénosides (C-hétérosides) & - & - \\
\hline Anthracénosides (O-hétérosides) & - & + \\
\hline Flavonoïdes & +++ & +++ \\
\hline Saponosides & +++ & +++ \\
\hline Tanins(FeCl) & +++ & +++ \\
\hline Tanins HCl concentré) & ++ & + \\
\hline Tanins (galliques) & - & - \\
\hline Tanins cathéchiques & ++ & +++ \\
\hline Composés réducteurs & - & - \\
\hline Oses et holosides & +++ & ++ \\
\hline Mucilages & ++ & - \\
\hline Stérols et triterpènes & ++ & + \\
\hline Hétérosides cardiotoniques (R. & - & + \\
\hline Marthoud) & & - \\
\hline Hétérosides cardiotoniques(Kedde) & - & - \\
\hline Hétérosides cardiotoniques(Baljet) & - & - \\
\hline Anthocyanes & - & +++ \\
\hline Leucoanthocyanes & +++ & \\
\hline
\end{tabular}

- : pas de coloration +++ : coloration intense ++ : coloration moyenne + : faible coloration

Le screening phytochimique a révélé la présence des coumarines, des tanins catéchiques, des stérols et triterpènes, des flavonoïdes, des saponosides, des mucilages, des oses et holosides, des anthocyanes et des leucoanthocyanes dans A. hispidum.

Wilfrid et al., 2015 ont rapporté la présence des tanins galliques et catéchiques, des flavonoïdes; des anthocyanes, des leucoanthocyanes, des saponosides, des terpenoides et stéroïdes, des mucilages et des saponines dans les feuilles de A. hispidum, ce qui confirme cette étude qui concerne la partie aérienne de la plante.

Kheraro et Adams (1974), d'une part et d'autre part Wilfrid et al. 2015 ont rapporté la présence d'alcaloïdes dans A. hispidum. 
Comme Diarra 2006, nous n'avons décelé ni alcaloïde, ni hétéroside cardiotonique, ni caroténoïdes et ni composés réducteurs dans l'échantillon qui a fait l'objet de cette étude.

Dans $X$. americana nous avons noté la présence des coumarines, des tanins cathéchiques, des stérols et triterpènes, des saponosides, des flavonoïdes, des hétérosides cardiotoniques, des oses et holosides, des génines de flavonoïdes, des o-hétérosides et des leucoanthocyanes. Ces résultats corroborent avec ceux de Keita, 2004, Soro et al., 2015 Roumana et al., 2015, et Zeinab, 2016.

\section{Teneur en polyphénols totaux}

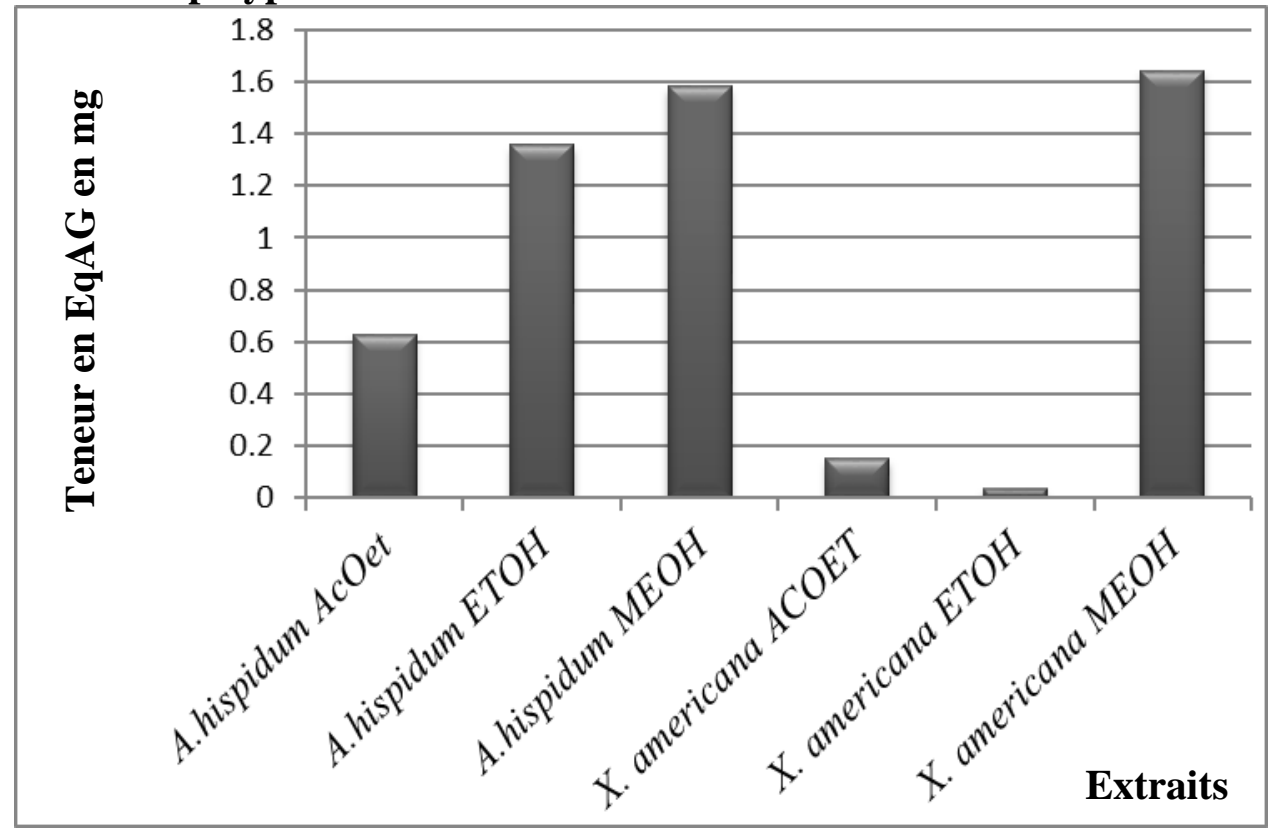

Figure 2: Teneur en polyphénols totaux

Les teneurs en polyphénols totaux des différentes fractions varient entre 0,627 et $1,583 \mathrm{mg}$ d'équivalent acide gallique (EqAG) pour A. hispidum et de 0,036 à 1,645 d'EqAG pour X. americana. La teneur en polyphénols totaux la plus élevée a été mesurée dans l'extrait méthanolique de $X$. americana, avec une teneur en EqAG de 1,645 mg, par rapport aux extraits AcOEt et EtOH, ou nous avons obtenu des teneurs de l'ordre de $0,627 \mathrm{mg}$ et $1,358 \mathrm{mg}$ EqAG/g pour $A$. hispidum et 0,151 à 0,036 pour $X$. americana respectivement (Figure 2).

D'après ces résultats, on déduit que la teneur en polyphénols totaux dans les extraits dépend de la polarité du solvant utilisé pour l'extraction. 


\section{Teneur en flavonoïdes}



Figure 3: Teneur en flavonoïdes totaux

Les teneurs en flavonoïdes des extraits d'A. hispidum et $X$. americana ont été calculées à partir de la courbe d'étalonnage tracée en utilisant la quercétine comme standard. Elles sont exprimées en équivalent quercétine (EqQ).

Les résultats présentés sur la figure 3 montrent que les teneurs en flavonoïdes totaux varient considérablement en fonctions du solvant d'extraction de l'échantillon. A. hispidum enregistre un maximum de teneur en flavonoïdes avec l'extrait AcOet avec $0,089 \mathrm{mg} / \mathrm{EqQ}$ alors que pour $X$. americana la teneur maximale a été obtenue avec l'extrait EtOH avec 0,069 $\mathrm{mg}$ en EqQ. L'extrait méthanolique, 0,613 mg EQ/g, suivi de l'extrait hexanique qui renferme des teneurs plus faibles, 0,148 mg EQ/g (Figure2).

\section{Activité Antioxydante}

L'activité antioxydante des extraits AcOEt, EtOH et $\mathrm{MeOH}$ de $A$. hispidum et $X$. americana a été quantifiée par spectrophotométrie. La figure 4 indique les résultats obtenus. 


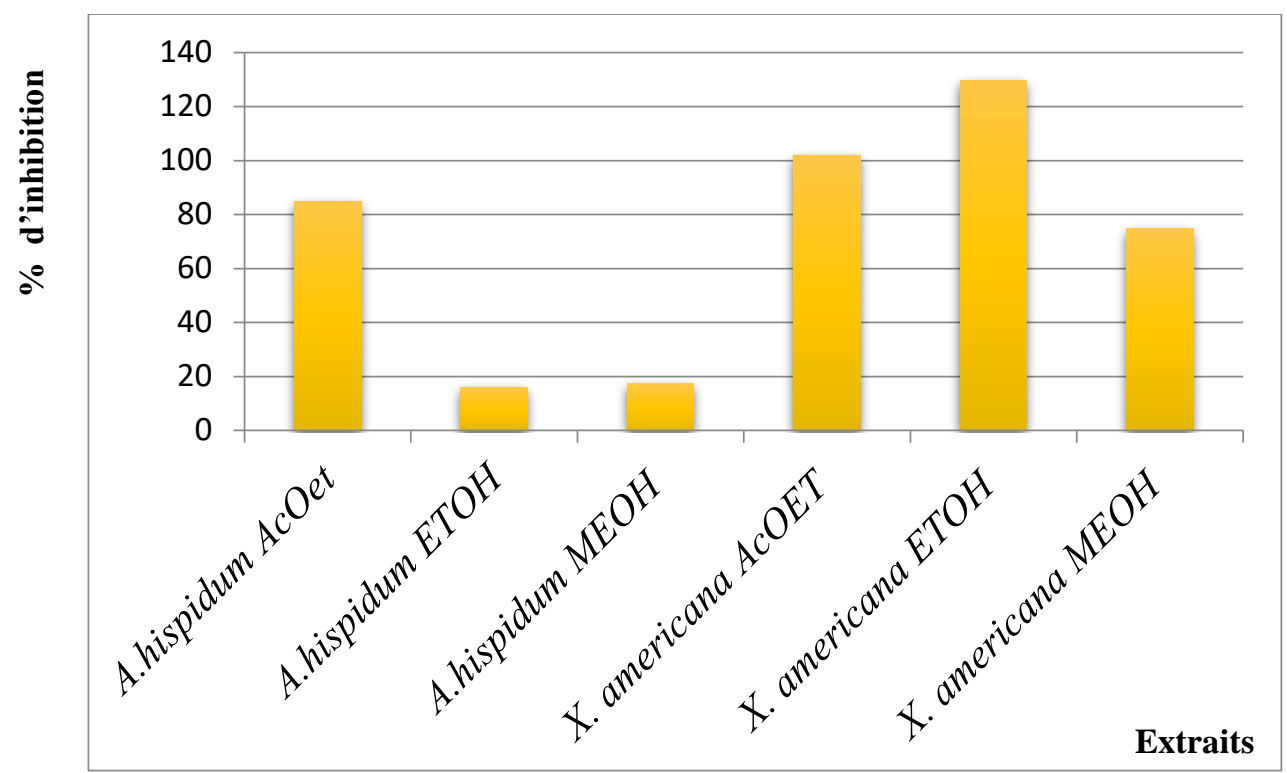

Figure 4 : Activité antioxydante des extraits de A. hispidum et X. americana

L'extrait EtOH de l'écorce de tronc de $X$. americana a montré la plus grande capacité antiradicalaire de $129,56 \%$ due à sa forte teneur en flavonoïdes (Figure 3) et en composés phénoliques totaux (Figure 2), l'activité antioxydante des extrait de $X$. americana et d'A. hispidum pourrait être due à la forte teneur en acide gallique (Figure 4); en flavonoïdes et en tanins des extraits.

L'augmentation de la consommation de produits dérivés des substances naturelles dans l'alimentation quotidienne est associée à une diminution du risque de mortalité dans les maladies coronariennes, les accidents vasculaires cérébraux et les dommages secondaires à l'hypertension artérielle (Akram et $\mathrm{BiBi}, 2014$ ).

De nombreuses études ont montré que les polyphénols pouvaient exercer un effet bénéfique sur le système cardiovasculaire tels que des effets anti-athérothrombotiques (Auger et al., 2002; Wollny et al. 1999), une inhibition de l'adhésion et de l'agrégation plaquettaires (Wollny et al.,1999; Freedman et al., 2001), l'oxydation des lipoproteines (Frankel et al. 1993) et de la prolifération et de la migration des cellules musculaires lisses prévenant ainsi le remodelage vasculaire (Iijima, 2000, 2002). L'effet bénéfique des polyphénols sur la santé cardiovasculaire a été attribué en partie à leur effet direct sur les vaisseaux sanguins, plus particulièrement sur l'endothélium (Martin et Andriantsitohaina, 2002; Rosario et al. 2007; Monica et al., 2010; Rafael et al., 2012; Cyril et Valérie 2014; Dina et al., 2019). Des études expérimentales et cliniques ont révélé que les polyphénols sont capables d'augmenter la formation endothéliale de facteurs vasoprotecteurs comme le 
monoxyde d'azote (NO).Ce dernier un est puissant vasodilatateur, inhibiteur de réponses pro-inflammatoires et pro-thrombotiques, améliore la dysfonction endothéliale et le stress oxydant vasculaire qui contribuent au développement des pathologies cardiovasculaires majeures comme l'hypertension artérielle (Auger et Valérie, 2014). De plus, les polyphénols peuvent également exercer un effet protecteur vasculaire en agissant directement sur le vaisseau sanguin principalement en améliorant les mécanismes vasoprotecteurs dépendants de l'endothélium, comme la formation endothéliale de NO et l'induction de l'hyperpolarisation dépendante des cellules endothéliales.

Les polyphénols peuvent exercer leur effet cardioprotecteur par activation de nombreuses puissantes voies cellulaires impliquant les intermédiaires métaboliques, les microARN et les sirtuines (Lecour et Lamont, 2011).

Macha et Mustafa (2005), suggèrent que le traitement chronique par les flavonoïdes (baicalein, flavone, et quercétine) préserve les fonctions endothéliales vasculaires des animaux hypertensifs par le biais de plusieurs actions possibles, notamment l'augmentation de la production et de la biodisponibilité de l'oxyde nitrique endothélial et la réduction de la pression sanguine.

Ning et al., (2005) ont rapporté que l'ingestion des polyphénols de pépins de raisin diminuent la pression des rats spontanément hypertensifs, probablement via un mécanisme antioxydant. Les flavonoïdes servent de médiateurs avec ses effets antihypertenseurs par l'augmentation de l'oxyde nitrique (NO), la biodisponibilité, la réduction du stress oxydatif des cellules endothéliales ou moduler l'activité des canaux ioniques vasculaires (Dina et al., 2019)

\section{Conclusion}

Le screening phytochimique de la partie aérienne de A. hispidum et l'écorce de tronc de $X$. americana a révélé la présence de stéroïdes, terpenoides, flavonoïdes, tanins, saponines et glycosides dans ces drogues.

La quantification de composés polyphénoliques totaux et flavonoïdes, et la détermination de l'activité antioxydante par spectrophotométrie dans les extraits de nos A. hispidum et de X. americana a permis de déduire que l'utilisation récurent de ces plantes serait lié à leur richesse relative en constituants polyphénoliques.

Les observations ci-dessus suggèrent que la partie aérienne de $A$. hispidum et l'écorce de tronc de $X$. americana contiennent des molécules qui pourraient justifier leur utilisation dans la prise en charge traditionnelle de l'hypertension artérielle. 


\section{References:}

1. Akram K, BiBi LH (2014), Phytochemicals and Hypertension. Shiraz E-Medical Journal; 15(1):e19738.

2. Auger C, Valérie BS, (2014). Potentiel des polyphénols à améliorer la protection vasculaire en stimulant la fonction endothéliale, Cahiers de nutrition et de diététique 49, 160-172.

3. Assogba P, Dansou H. and Joachim D. Gbenou, (2015). Diuretic and natriuretic activities from ten medicinal plants used in south Benin, Journal of Chemical and Pharmaceutical Research,7(12):1145-1152.

4. Brand-Williams W, Cuvelier ME, Berset C, (1995).Use of a free radical method to evaluate antioxidant activity, Lebensmittel Wissenschaft \& Technologie., 28, 25-30.

5. Bruneton J (1999). Pharmacognosie : Phytochimie, plantes médicinales. Technique \&documentation-Lavoisier,1120p.

6. Cicco N, Lanorte MT, Paraggio M, Viggiano M, Lattanzio V (2009). A reproducible,rapid and inexpensive Folin-Ciocalteu micro-method in determining phenolics of plant methanol extracts. Microchemical Journal 91, 107-110.

7. Cyril A, Bertrand C, Nicolas L, Pierre LT, Caroline L, Gerard C, Pierre B, Jean-Max R (2002). Red wine phenolic compounds reduce plasmalipids and apolipoprotein $\mathrm{B}$ and prevent early aortic atherosclerosis in hypercholesterolemic golden Syrian hamsters (Mesocricetus auratus). The Journal of Nutrition, 132(6):1207-13.

8. Diarra Y, (2006). Etude phytochimique et des activités biologiques de Acanthospermum hispidum et de Curiculigo pilosa schun et Thonn deux plantes utilisées dans le traitement traditionnel de l'hypertrophie de la prostate (HBP) .Thèse de pharmacie, Bamako. FMPOS, Bamako $143 p$.

9. Dina M, Abdullah AS, Gianfranco P, Ahmed E and Ali HE (2019). Flavonoids in hypertension: a brief review of the underlying mechanisms. Current Opinion in Pharmacology, 45:57-65.

10. Dirar AI, Alsaadi DHM, Wada, Mohamed MMA, Watanabe T, Devkota HD, 2019. Effects of extraction solvents on total phenolic and flavonoid contents and biological activities of extracts from Sudanese medicinal plants. South African Journal of Botany 120, 261-267.

11. Evans WC. (2002). Pharmacognosy. Saunders Elsevier 585p.

12. Freedman JE, Parker III C, Li L, Perlman JA, Frei B, Ivanov V, et al., (2001). Select flavonoids and whole juice from purple grapesinhibit platelet function and enhance nitric oxide release.Circulation; 103(23):2792-8. 
13. Frankel EN, Kanner J, German JB, Parks E, Kinsella JE, (1993), Inhibition of oxidation of human low-density lipoprotein by phenolic substances in red wine. The Lancet, 341 (8843): 454-457.

14. Gönenç A, Hacışevki A, Tavil Y, Çengel A, Torun M., (2013). "Oxidative stress in patients with essential hypertension: a comparison of dippers and nondippers", European Journal of Internal Medecine, 24: $139-144$

15. Hassimi L, Bako H., Boubacar A., Toure AI, (2013). Hypertension artérielle chez les hémodialysés chroniques à l'hôpital national lamordé de Niamey. Mali medical $\mathrm{N}^{\circ} 4$ : 37-42

16. Helmut MH, Neale J, Brian M, Anthony LZ, Charlie CX (2016). Polyphenol protection and treatment of hypertension. Phytomedicine 220-231.

17. Hertog MG, Hollman PC, Katan MB, Kromhout D. (1993).Intake of potentially anticarcinogenic flavonoids and their determinants in adults in The Netherlands. Nutrition and Cancer; 20:21-29.

18. MG, Feskens EJ, Hollman PC, Katan MB, Kromhout D. (1993). Dietary antioxidant flavonoids and risk of coronary heart disease: the Zutphen Elderly Study. Lancet, 342: 1007-1011.

19. Iijima K, Yoshizumi M, Hashimoto M, Akishita M, Kozaki K, AkoJ, et al. (2002). Red wine polyphenols inhibit vascular smooth muscle cell migration through two distinct signaling pathways. Circulation, 105(20):2404-2410.

20. Iijima K, Yoshizumi M , Hashimoto M, Kim S, Eto M, Ako J, et al. (2000). Red wine polyphenols inhibit proliferation of vascular smooth muscle cells and down regulate expression of cyclin A gene. Circulation, 101(7):805-11.

21. Inbathamizh L, Ponnu TM, Mary EJ (2013). "In vitro evaluation of antioxidant and anticancer potential of Morinda pubescens synthesized silver nanoparticles", Journal of Pharmaceutical Research.; 6:3238.5 .

22. Juanying O, Mingfu W, Jie Z, Shiyi O(2019). Positive and negative effects of polyphenol incorporationinbaked foods. Food chemistry. 284, 90-99.

23. Kannika S, Woranan N, Michael H, Nitra N (2018). Effect of drying methods and solvent extraction on the phenolic compoundsof Gynura pseudochina (L.) DC. leaf extracts and their anti-psoriatic property. Industrial Crops \& Products 120, 34-46.

24. Keita A., (2004). Etude de trois plantes utilisées dans le traitement traditionnel de l'ulcère gastro-duodénal dans le District de Bamako: Borassus athiopum Mart (Palmeae), Sclerocarya birrea (A.Rich.). 
Hochst. (Anacardiaceae) et Ximenia americana L. (Olacaceae).Thèse de pharmacie. Bamako. FMPOS, Bamako.198p.

25. Kerharo J. et Adams J. G. (1974). La pharmacopée sénégalaise traditionnelle plantes médicinales et toxiques. Edition Vigot et Frères, Paris, $1011 \mathrm{p}$.

26. Lecour S and Lamont KT (2011), Natural Polyphenols and Cardioprotection. Mini-Reviews in Medicinal Chemistry, 11, 11911199.

27. Leong LP, Shui G., (2002). An investigation of antioxidant capacity of fruits in Singapore markets. Food Chemistry 76: 69-75.

28. Lobo V, Patil A, Phatak A, Chandra N. (2010). "Free radicals, antioxidants and functional foods: impact on human health", Pharmacognosy Reviews., 4:118-126.

29. Machha A, Mustafa MR, (2005). Chronic treatment with flavonoids prevents endothelial dysfunction in spontaneously hypertensive rat aorta. Journal of Cardiovascular Pharmacology; 46(1): 36-40.

30. Martin S et Andriantsitohaina R (2002), Mécanismes de la protection cardiaque et vasculaire des polyphénols au niveau de l'endothélium. Annales de cardiologie et d'angéiologie 51, 304-315

31. Ministère de la Santé Publique (MSP) (2008). Enquête sur la Mesure des facteurs de risque des maladies non transmissibles au Niger (Approche Step"wise" de l'OMS).

32. Mohi E.M. A. Z. (2016). Isolation and Characterization of Some Constituents of Ximenia Americana. L Bark. Thesis for the Fulfillment of the Requirements for the Degree of Doctor of Philosophy in Chemistry. Sudan University of Science and Technology. 93p.

33. Middleton Jr E, Kandaswami C, Theoharides TC, (2000). The effects of plant flavonoids on mammalian cells: implications for inflammation, heart disease, and cancer. PharmacologgyReview;512:673-675

34. Monica Galleano, Olga Pechanovab and Cesar G. Fraga (2010), Hypertension, Nitric Oxide, Oxidants, and Dietary Plant Polyphenols. Current Pharmaceutical Biotechnology, 11, 837-847.

35. Ning P, John TC, Jeevan P, Helen K, Roger WC, Michael WJ (2005). Antihypertensive and cognitive effects of grape polyphenols in estrogen-depleted, female, spontaneously hypertensive rats. American Journal Physiology Regulatory Integrative and Comparative Physiology289(3):R771-R775.

36. Rafael M, Rocio M, Maria LM, Alzenira F, Luis J, Antonio J V, Francisco JGM, Jose V and Pablo S (2012). Olive Oil Polyphenols Decrease Blood Pressure and Improve Endothelial Function in Young 
Women with Mild Hypertension. American journal of hypertension, volume $25 \mathrm{~N}^{\circ} 12,1299-1304$.

37. Ramana KV, Srivastava S, Singhal S., (2013). "Lipid peroxidation products in human deathanddisease" Oxidative Medecine and Longevity 1-3.

38. Rosario J, Rocío L, María K, Miguel R, Rocío V, Manuel S, Félix V, Francisco O, Antonio Z (2007), Polyphenols restore endothelial function in DOCA-salt hypertension: Role of endothelin-1 and NADPH oxidase. Free Radical Biology \& Medicine, 43462-473.

39. Roumanatou SN, Ibrahim ML, Moussa I, Mahamadou B, Ilagouma AT, Abdoulaye A, Ouwe MO., Ikhiri K (2015). «Évaluation in vitro de l'activité antiplasmodiale d'extraits de plantes issues de la pharmacopée traditionnelle du Niger ». Journal of Applied Biosciences 89:8291-8300,doi:10.4314/jab.v89i1.8

40. Soro TY, Néné-bi AS, Zahoui OS, Yapi A. et Traoré F. (2015). Activité anti-inflammatoire de l'extrait aqueux de Ximenia americana L. (Olacaceae). Journal of Animal \& PlantSciences.Vol.24, Issue 3: 3802-3813.

41. Wilfrid KDD. Agbodjogbe, Judith F. Ahounou Aïkpe, Marc Abel Ayedoun, Fidèle M. traditionnel de l'hypertrophie de la prostate (HBP) .Thèse de pharmacie, Bamako. FMPOS, Bamako143p.

42. Wollny T, Aiello L, Di TD, Bellavia V, Rotilio D, Donati MB, et al. (1999). Modulation of haemostatic function and prevention of experimental thrombosis by red wine in rats: a role for increased nitric oxide production. British Journal of Pharmacolology; 127(3):747-755.

43. Zeinabou MTA, Madi N, Yaya K, Moumouni G, Ahmet TL, Hassane DM, Idé AEC Magni Yogo (2016). Insuffisance Rénale Aigue Obstétricale : Expérience De La Maternité Issaka GAZOBY de Niamey (Niger) European Scientific Journal. edition vol.12, $\mathrm{N}^{\circ} .33$ : 1857-7881

44. Zerargui F, Boumerfeg S, Charef N, Baghiani A, Djarmouni M, Khennouf S, Arrar L, Musa H. Abu Zarga, Mohammad SM. (2015). Antioxidant Potentials and Xanthine Oxidase Inhibitory Effect of Two Furanocoumarins Isolated from Tamus communis L. Medicinal . Chemistry; 11:506-513. 